\title{
Research on Modern Office Information System under Cloud Environment
}

\author{
Yu Mingyi \\ Chongqing College of Electronic Engineering, Chongqing, China
}

Keywords: Cloud Environment, Modern Office Information, Computer Technology

\begin{abstract}
Cloud computing is the art Internet technology in the current stage, with large scale, resource mobility, virtualization, economy and so on. By applying cloud computing technology in office systems, the function has been significantly improved. Office information system involves the economic, management and other professional contents, which also put forwards higher requirements for computer technology. Office information system needs to coordinate various resources and subject characteristics, and then solves complicated work through the simple and easy-to-use office interface. With the gradual acceleration of social development, office information systems should also be constantly adapted to the current situation of development and solve more office problems. This also poses a greater work challenge for computer technology developers. Modern office information system covers almost all aspects of day-to-day office work, including document processing, online conferences, peer-to-peer instant messaging and so on. In the whole office information system, the main hardware facilities are computers, and the software part is installed in the corresponding office software, people can open the office in any corner of the world electronic. Office information system mainly through computer information technology, daily office documents, images, audio, video and other contents is converted to multimedia data for successful transmission. Therefore, in order to ensure the work efficiency of the office information system, computer developers need to adopt the development standard, and make the system run efficiently under the premise of ensuring the information security so as to achieve the purpose of improving office work efficiency.
\end{abstract}

\section{Introduction}

With the continuous development of network information technology, in the aspect of office electronicization, modern office information systems with higher technology contents have become the effective assistant of people's office. The office information system can not only bring more efficient support to people's work, it can also help people make decisions from a higher perspective. With the development of various application software, the computer technology has made great progress in the office information system. Computer science and technology issues in the office information system research is also deepening, the author based on the current social development, the office system in computer science and technology to do a brief discussion [1].

Cloud computing is a new Internet technology developed in recent years, and has been widely used in the fields of network storage, network security and online games. The technology is based on the Internet to add, use and deliver services related services, often involving the provision of dynamically scalable and often virtualized resources over the Internet. In this way, shared hardware and software resources and information are allowed to be provided to a computer or other device as required. Typical cloud computing uses, for example, users can use a commercial application over the network, and the application's software, data are stored on the cloud server. Cloud computing has the following characteristics. "Cloud" is very large, providing cloud service companies to invest hundreds of servers for cloud computing, as shown in Figure 1 strong computing power to ensure the mass data communication / storage system efficient and stable operation, to meet the client's massive data Interaction. Cloud computing can be built on top of different underlying platforms that can effectively accommodate a wide range of different types of hardware and software infrastructure resources. Users in any location, the use of a variety of terminals can access application services, no fixed physical entities, the computer can, mobile phones can be. And in the 
"cloud" under the support of the ever-changing applications can be constructed, the same "cloud" can support different applications at the same time. The transportation resources are dynamic and can be distributed on demand. The cloud computing platform can realize the isomorphism and quantification of computing resources through the virtual splitting technology. The centralized and centralized management of the cloud greatly improves the resource utilization rate of the users, the cost of cloud computing on-demand users to reduce the cost [2].

\section{Cloud Office and the Traditional Office System Comparison}

Compared with the cloud office system, the traditional office system on the client and configuration have certain requirements. Most of the traditional office software needs to be run on the computer side, occupying a large space on the computer side, the mobile terminal can not have the operating conditions of the traditional office software, and the capability of cross-platform is weak so that the requirements of light weight and fragmentation of the modern office can not be realized. Moreover, the ordinary office software is run independently, does not support the office team members work together. The traditional office software that supports this function is more expensive, complicated to maintain, and difficult to popularize applications. Such as the most widely used office software, computer hardware has certain requirements, each computer using the software needs to download and install client software. Weak cross-platform capabilities, i OS, Android and other new smart operating systems do not have enough support. But also "one person, one software", it is difficult to achieve coordination office. Cloud office system compatible with a variety of document formats, coupled with its powerful information processing capabilities, can be analyzed in a comprehensive form required to show to different users, and both support multiple accounts of the collaborative office and online office [3].

Cloud office system is relying on the "cloud" operation, you need to work in the Internet environment. Therefore, compared with the traditional office system, the cloud office system relies on a stable network environment and has higher security requirements. In addition, the cloud office system can achieve a lot of powerful functions, the requirements of the office system itself are also higher, and the office system used in the cloud environment is easier to use and easier to operate than the ordinary system.

\section{Cloud Technology in Office Information System}

Since the start of the application of computer technology exclusive application is not very early in our country, since the implementation of the network TCP / IP connection index, the nationwide computer information network project started to start freely and began to provide all kinds of information accurate inquiry to the grass-roots community. E-mail Fast sending and receiving a number of business. It should be noted that after a long period of practice investigation, technical personnel often encounter the following unexpected problems during ISP implementation. First, the selection of service trunks is not sophisticated enough, which often results in a lot of time and energy wasted by the office staff during log-in. Second, different ISP units in the corresponding service charges and network usage billing are all on the distinction, the need for the main office in the early use of more audit.

With the large variety of communication software provided within the office market in our country, any flaws in the layout will cause the existing interface to be not friendly enough. Furthermore, because the internal resource and equipment configuration environment is too complicated, many office personnel computers The use of skills is low, if the company does not carry out a certain amount of computer operation training, some of the functions of the office information system will be difficult to play a role in practical work [4].

At present, such programs have provided auxiliary functions for office automation systems, including high-speed fax transmission, flexible reception, and EDI functions. First of all, in the fax sending function debugging level, the main is the application of Windows programs for Word, 
Excel documents sent directly, and additional fax cover summary information; followed by fax receiving function control level, in fact, the fax function to run in the background, you can proceed Other programs operate while receiving faxes. When there is a phone cut in, the staff can also manually cut in and out of the way to send and receive faxes. At the EDI debugging level, EDI functionality is an efficient and easy way to exchange remote files without being connected to the network. Files transferred can be spreadsheets, WOERD or other formatted text, or even applications.

\section{Cloud Computing Technology in the Modern Office System}

Cloud computing can share information to the cloud, and everyone in the office team can contribute that resource over the Internet, saving time and money compared with traditional editing. The resources shared among them include not only the resources shared by the staff inside the office team, but also the external information that is allowed to be shared on the cloud server through the interface. This way of resource sharing not only can effectively utilize the resources within the team, but also boosts the efficiency of the office with external information. Most enterprises applying the traditional office system will set up multiple data centers. Information sharing and scheduling in different data centers have certain limitations, and the sharing and scheduling functions are more complicated. According to the statistics of resource sharing degree of each business system in the traditional office software, its utilization rate is within $30 \%$, and through cloud computing, this value can be increased to $60 \%$. Thus, in terms of resource sharing and resource utilization, the application of cloud computing in modern office systems has achieved remarkable results [5].

Cloud office applications have a strong synergy, its powerful cloud storage capabilities not only make data documents ubiquitous, more in line with new concepts such as cloud communications, around the document for intuitive communication and discussion, or collaborative editing of many people, thereby greatly improving teamwork Project efficiency and quality. Take a team that provides APP development services as an example. The project leader submits the tasks to the cloud office system. Product managers, page designers and software engineers will schedule tasks into the work plan. Product manager according to customer needs and ideas given development, spread to the cloud, page designers and software engineers to start the design and development, and the results of the stage to share in the cloud, the three parties can view APP development status anytime, anywhere and can be modified The work of all parties can adjust the work content according to the suggestion at any time. Cloud office system powerful information processing capabilities and support for a variety of terminal features make the team easier to communicate and collaborate. Cloud computing virtualization allows the cloud office system can take advantage of smart devices as a carrier cloud office application can help customers record and modify the contents of the document at any time, and synchronized to the cloud storage space. The cloud office application allows users to use the same office environment regardless of the terminal device used. This enables the mobility and fragmentation of the office. The users of the cloud office system can access the office through the Internet at any time by using a variety of terminal devices.

\section{Conclusion}

With the accelerated pace of work nowadays, the powerful information processing capabilities of cloud office systems and the features that can be applied anytime and anywhere can better meet the needs of office workers. Through the Internet and a variety of electronic products, cloud office system can achieve resource sharing, collaborative office, online office, optimize office processes, significantly improve team productivity. The continuous development of computer science and technology in our country, its field of application is also more and more widely, especially the office information system is represented. The author believes that in an increasingly fierce market competition, companies want to get greater development and upgrading, in the market place, we must strengthen the office information system to transform, consolidate and enhance the overall 
efficiency, strengthen the aspects of Attention and attention.

\section{References}

[1] Yang Bin. Cloud computing-based virtual technology in business information system [J]; Information Technology Research, 2014,2: 1-4.

[2] Zhao Hui. Analysis of Office Automation System Based on Cloud Computing [J]; Computer Knowledge and Technology (Academic Exchange), 2014,1: 231-232.

[3] Yu Hua. Intelligent terminal virtualization technology will drive the trend of office mobility [J]; Economic Perspective, 2016,15: 144.

[4] Xu Ying. Application of computer information processing technology in office automation [J]. Information Engineering, 2016 (11): 32-33.

[5] Tang Zhuoqun. Research on Problems Existing in the Development of Computer Science and Technology [J]. Information Systems Engineering, 2017 (08): 12-12. 